\title{
Antibiotic Resistance and Trend of Urinary Pathogens in General Outpatients from a Major Urban City
}

\author{
Carlos R. Kiffer, Caio Mendes, Carmen P. Oplustil, Jorge L. Sampaio \\ Section of Microbiology, Fleury Institute, Sao Paulo, SP, Brazil
}

\begin{abstract}
Objective: We assessed the antimicrobial resistance patterns of pathogens responsible for urinary tract infections (UTI) in outpatients in São Paulo, Brazil, as well as the Escherichia coli antimicrobial resistance trend.

Materials and Methods: Outpatients urine cultures were collected from January 2000 to December 2003. Statistical analysis considered positive results for one bacterial species with colony count $\geq 100,000 \mathrm{CFU} / \mathrm{mL}$. Stratification was done on age group and gender. Statistical tests used included chi-square and the chi-square test for trend to evaluate differences between susceptibility rates among age groups and ordering in the E. coli resistance rates per year, respectively.

Results: There were 37,261 positive results with Enterobacteriaceae isolated in 32,530 (87.3\%) and Gram-positive cocci in $2,570(6.9 \%)$ cultures. E. coli had the highest prevalence (71.6\%). Susceptibility tests were performed in 31,716 cultures. E. coli had elevated resistance rates (> 30\%) to ampicillin, trimethoprim-sulfamethoxazole, and tetracycline. Significant differences between age groups and ordering among years were observed.

Conclusions: The use of trimethoprim-sulfamethoxazole is precluded in the population studied due to elevated resistance rates (> 30\%) among most prevalent pathogens. Significant resistance rate differences among age groups and years were observed, particularly for fluoroquinolones. Fluoroquinolones should be used with caution. Nitrofurantoin should be used as empirical therapy for primary, non-complicated urinary tract infections.
\end{abstract}

Key words: urinary tract infections; drug therapy; drug resistance; Escherichia coli

Int Braz J Urol. 2007; 33: 42-9

\section{INTRODUCTION}

Controlling the increase in antimicrobial resistance is a major issue confronting organized health care today. Although multiple factors play a role in this problem, the selective pressures of inappropriate and widespread use of antibiotics are considered major contributors. A few studies have analyzed the antimicrobial resistance patterns of bacteria causing community acquired urinary tract infections (UTI) (1-5). International resistance surveillance studies have shown an increasing resistance pattern against com- monly used community antimicrobials (1-6). Surveillance programs may be valuable tools and may offer important information on bacterial resistance trends either per geographical location or per disease type in community or hospital settings $(7,8)$. However, surveillance programs must be stratified by region and population, not to incur in general and mostly nonapplicable conclusions. Regional studies analyzing community acquired UTI and their antimicrobial resistance pattern are currently needed in many areas, particularly major urban centers worldwide because of their specificities on antimicrobial usage density. 
Thus, the goal of this study was to assess the most frequent pathogens responsible for urinary tract infections (UTI) in outpatients and their antimicrobial resistance pattern in São Paulo, Brazil, through consecutive urine samples collected during a fouryear period, from January 2000 to December 2003. Additionally, the study also aimed at identifying possible resistance trends.

\section{MATERIALS AND METHODS}

From January 2000 to December 2003, routine urine cultures were consecutively collected at a private medical diagnostic center from patients residing in the São Paulo city area, Brazil. The city is located in Southeast Brazil and has around 10,000,000 inhabitants. The private medical diagnostic center has 12 collection sites distributed over the city area and serves mostly the population with access to private health care system within this area. All cultures were collected from outpatients with medical requests through midstream urine samples, except for children below 2 years old, who had collections by sterile collector vials. More than one culture from the same patient was only included in the database if collected more than 30 days apart.

Urine samples were plated on cystine-lactoseelectrolyte-deficient (CLED), MacConkey and citrate agars (deep slide methodology) up to 20 minutes after collection and then incubated at $37^{\circ} \mathrm{C}$ for 18 to 24 hours. Negative urine samples (refrigerated aliquot) after initial 24 hours incubation with abnormal leukocyte count $(\geq 30,000 / \mathrm{mL})$ or bacteriuria on direct non-centrifuged urine microscopic examination were also cultivated on blood agar and incubated for an additional 24 hours.

Identification of all isolates was done by VITEK (bioMérieux Inc., Durham, North Carolina, USA) automated system. Susceptibility testing was performed according to medical requests and was done by VITEK (bioMérieux, Inc.) automated system for Enterobacteriaceae and disk diffusion test (Oxoid, Basingstoke, Hampshire, UK) were used for Grampositive bacteria and Pseudomonas aeruginosa. The following antimicrobials were tested against Gram-nega- tive isolates: ampicillin, ceftriaxone, cephalothin, ciprofloxacin, gentamicin, nalidixic acid, nitrofurantoin, norfloxacin, tetracycline, and trimethoprimsulfamethoxazole. The following antimicrobials were tested against Gram-positive isolates: ciprofloxacin, clindamycin, erythromycin, nitrofurantoin, oxacillin, penicillin G, tetracycline, trimethoprimsulfamethoxazole, and vancomycin (results not fully shown). Interpretative criteria used were for the respective years CLSI (formerly NCCLS) documents (9-12).

Only positive results with one bacterial species and a colony count $\geq 100,000 \mathrm{CFU} / \mathrm{mL}$ were considered for descriptive and inferential analysis. Stratification by gender and age was done, and age groups were divided as following: $<4$ years-old; $\geq 4$ and $<13$ years-old; $\geq 13$ and $<60$ years-old; and $\geq$ 60 years-old. In order to investigate possible factors associated to resistance, E. coli, as the most prevalent microorganism, and its susceptibility rates to ampicillin, ciprofloxacin, nitrofurantoin, norfloxacin, tetracycline, and trimethoprim-sulfamethoxazole were defined as the dependent variables. Age groups were defined as the independent variables. The chisquare test was used to identify differences between the observed E. coli susceptibility rates of the six antimicrobials among all four age groups. Additionally, the Fisher exact test was used to evaluate differences between the observed resistance rates between $<13$ and $\geq 60$ years old groups (13). An exact 2 tailed $\mathrm{P}$ value was computed, as well as the confidence intervals around the differences using the normal approximation. Finally, the chi-square test for trend, as described by Altman 1991 (13), was applied to compare ordering between E. coli resistance rates to ampicillin, ciprofloxacin, nitrofurantoin, norfloxacin, tetracycline, trimethoprimsulfamethoxazole, and nalidixic acid among the four years (2000 to 2003). P values below 0.05 were considered significant for all tests.

\section{RESULTS}

\section{Frequency of Microorganism Isolation}

There were 37,261 positive results and Enterobacteriaceae were isolated in $32,530(87.3 \%)$ cul- 
tures, followed by Gram-positive cocci with 2,570 $(6.9 \%)$. E. coli presented the highest prevalence (71.6\%), followed by Klebsiella pneumoniae $(6.4 \%)$, Proteus mirabilis (6.1\%), and Enterococcus faecalis (4.8\%), Pseudomonas aeruginosa (1.8\%), Staphylococcus saprophyticus (1.6\%), Enterobacter aerogenes $(1.6 \%)$, Enterobacter cloacae $(1.1 \%)$, and others $(5.0 \%)$.

Among the positive cultures, $88.8 \%$ belonged to female and $11.2 \%$ to male patients. Among the 33,090 UTI in females, $59.2 \%$ was detected in the population of $\geq 13$ and $<60$ years old. On the other hand, among the 4,171 UTI in males, $54.2 \%$ was detected in the population of $\geq 60$ years old. The age group of $<4$ years old contributed with $13.0 \%$ of cases in the male and with $4.4 \%$ in the female population.

Table- 1 shows the total amount of microorganisms isolated per age group and gender. Overall, E. coli was the most prevalent isolate in all groups. K. pneumoniae, although with a significant prevalence, was more commonly isolated in the population $\geq 13$ years old. P. mirabilis was more prevalent in the population $\geq 13$ years old, although the population $<4$ years old also presented a significant prevalence.

\section{Susceptibility Patterns}

Susceptibility tests were performed in 31,716 cultures. The susceptibility pattern of the most preva- lent microorganisms isolated from UTI in outpatients is described in Table-2. E. coli presented resistance rate to ampicillin of $43.4 \%$, followed by $33.7 \%$ to trimethoprim-sulfamethoxazole and by $30.5 \%$ to tetracycline. Ceftriaxone was the most active agent against E. coli ( $99.7 \%$ susceptible), with an extremely low resistance rate $(0.3 \%)$. Also, E. coli presented low resistance rates to gentamicin and nitrofurantoin (3.0\% and 2.9\%, respectively). For K. pneumoniae, the second highest prevalent pathogen, significant resistance rates were noted to nitrofurantoin, tetracycline, trimethoprim-sulfamethoxazole and nalidixic acid $(21.2 \%, 19.8 \%, 17.7 \%$, and $15.2 \%$ respectively), with low resistance rates to ceftriaxone, gentamicin, and ciprofloxacin $(1.7 \%, 3.3 \%$ and $6.0 \%$, respectively). For P. mirabilis, the third most frequently isolated, considerable rates were observed only to trimethoprim-sulfamethoxazole and ampicillin $(21.5 \%$ and $18.9 \%)$, apart from the intrinsic resistances to nitrofurantoin and tetracycline.

\section{Statistics}

E. coli was the most frequently isolated pathogen in all age groups $(22,693)$ and the majority had susceptibility tests performed $(22,679)$. Table- 3 shows E. coli resistance rates to ampicillin, ciprofloxacin, nitrofurantoin, norfloxacin, tetracycline, trimethoprim-sulfamethoxazole and the chi-square

Table 1 - Total amount of microorganisms isolated from UTI in outpatients and their distribution according to age groups and gender, years 2000 to 2003, São Paulo, Brazil.

\begin{tabular}{|c|c|c|c|c|c|c|c|c|c|}
\hline \multirow[b]{3}{*}{ Microorganism } & \multirow[b]{3}{*}{ Total } & \multicolumn{8}{|c|}{ Age Group } \\
\hline & & \multicolumn{2}{|c|}{$<4 \mathrm{y}-\mathrm{o}$} & \multicolumn{2}{|c|}{$\geq 4<13 \mathrm{y}-\mathrm{o}$} & \multicolumn{2}{|c|}{$\geq 13<60$ y-o } & \multicolumn{2}{|c|}{$\geq 60$ y-o } \\
\hline & & $\mathbf{F}$ & $\mathbf{M}$ & $\mathbf{F}$ & M & F & $\mathbf{M}$ & $\mathbf{F}$ & $\mathbf{M}$ \\
\hline E. coli & 26,693 & 972 & 252 & 1172 & 77 & 15230 & 730 & 7329 & 931 \\
\hline K. pneumoniae & 2,391 & 75 & 24 & 76 & 6 & 1128 & 61 & 912 & 109 \\
\hline P. mirabilis & 2,266 & 308 & 183 & 226 & 111 & 829 & 28 & 476 & 105 \\
\hline E. faecalis & 1,803 & 14 & 6 & 55 & 8 & 665 & 82 & 649 & 324 \\
\hline P. aeruginosa & 672 & 20 & 23 & 10 & 9 & 35 & 50 & 234 & 291 \\
\hline S. saprophyticus & 606 & 0 & 1 & 6 & 2 & 566 & 7 & 20 & 4 \\
\hline E. aerogenes & 596 & 10 & 15 & 7 & 0 & 375 & 21 & 131 & 37 \\
\hline E. cloacae & 391 & 13 & 14 & 13 & 2 & 98 & 30 & 136 & 85 \\
\hline C. freundii & 193 & 8 & 5 & 1 & 0 & 24 & 4 & 109 & 42 \\
\hline S. epidermidis & 161 & 0 & 1 & 6 & 1 & 58 & 12 & 38 & 45 \\
\hline
\end{tabular}


Table 2 - Resistance pattern (\%) of the most prevalent microorganisms isolated from UTI in outpatients, years 2000 to 2003, São Paulo, Brazil.

\begin{tabular}{lrrrrrrrrrrr}
\hline Microorganism & Total & AMP & CFL & CRO & CIP & GEN & NAL & NIT & NOR & SXT & TET \\
\hline E. coli & 22,679 & 43.4 & 13.9 & 0.3 & 11.9 & 3.0 & 15.5 & 2.9 & 12.0 & 33.7 & 30.5 \\
K. pneumoniae & 2,059 & 100.0 & 7.6 & 1.7 & 6.0 & 3.3 & 15.2 & 21.2 & 8.9 & 17.7 & 19.8 \\
P. mirabilis & 1,944 & 18.9 & 3.1 & 0.4 & 4.2 & 2.3 & 8.9 & 100.0 & 4.0 & 21.5 & 100.0 \\
E. faecalis & 1,525 & 0.3 & - & - & 16.1 & - & - & 0.8 & - & - & 59.2 \\
P. aeruginosa & 605 & - & - & 89.1 & 63.4 & 48.3 & - & - & 61.6 & - & - \\
S. saprophyticus & 531 & - & - & - & 1.3 & - & - & 0.6 & - & 7.0 & 15.8 \\
E. aerogenes & 510 & 100.0 & 100.0 & 2.0 & 5.1 & 2.2 & 9.6 & 21.2 & 5.5 & 7.8 & 6.9 \\
E. cloacae & 331 & 100.0 & 100.0 & 32.9 & 38.7 & 24.2 & 44.4 & 39.6 & 41.1 & 38.4 & 47.0 \\
C. freundii & 168 & 100.0 & 100.0 & 20.2 & 22.6 & 12.5 & 28.6 & 8.3 & 21.4 & 25.6 & 53.5 \\
\hline
\end{tabular}

$-=$ Not tested $; A M P=$ ampicillin $; C F L=$ cephalotin $; C R O=$ ceftriaxone $; C I P=$ ciprofloxacin $; G E N=$ gentamicin $;$ NAL $=$ nalidixic acid $;$ NIT = nitrofurantoin $;$ NOR = norfloxacin $;$ SXT = trimethoprim-sulfamethoxazole $;$ TET $=$ tetracycline.

test with $\mathrm{P}$ values for comparisons of proportions among age groups.

Table-4 shows a test intended to identify possible ordered resistance rates of the most prevalent microorganism during the study period. The chisquare test for trend was performed for comparisons of the ordered differences between E. coli resistance rates to the described antimicrobials among the four years surveyed, i.e. 2000 to 2003.

\section{COMMENTS}

Uncomplicated UTI are amongst the most common infections in outpatient women with significant morbidity and health costs (14). This study did not discriminate among uncomplicated and complicated UTI in the sample evaluated and this could lead to a case mix with confounding variables in terms of resistance patterns. However, it should be noted that the vast majority of the samples collected from adults correspond to clean-catch midstream urine and that it is a common practice in our area to order urine cultures for uncomplicated UTI, since results are available no later than 48 hours after sample collection. Additionally, as stated, only one urine sample per patient in a 30-day period was entered in the database, diminishing the possibility of frequent UTI recurrences.

Although the spectrum of agents causing UTI in outpatients is relatively constant, their susceptibility patterns are different in different environments.

Table 3 - Chi-square test for comparisons of E. coli resistance rates (\%) to various antimicrobials among age groups.

\section{E. coli Resistance Rate (\%)}

\begin{tabular}{lrcccccc} 
Age Group & N & Ampicillin & Ciprofloxacin & Nitrofurantoin & Norfloxacin & Trimeth-sulfa & Tetracycline \\
\hline$<4$ & 969 & 60.8 & 0.9 & 1.9 & 1 & 49.6 & 33.7 \\
$\geq 4$ and $<13$ & 979 & 57.4 & 1.5 & 1 & 2.1 & 49.0 & 32.2 \\
$\geq 13$ and $<60$ & 13,675 & 39.8 & 7.1 & 1.6 & 7.2 & 29.8 & 26.6 \\
$\geq 60$ & 7,056 & 46.1 & 24.3 & 5.9 & 24.4 & 37.0 & 37.3 \\
Chi-square & & 209.61 & 1450.24 & 308.04 & 1424.65 & 271.37 & 217.34 \\
P value & & $<0.0001$ & $<0.0001$ & $<0.0001$ & $<0.0001$ & $<0.0001$ & $<0.0001$ \\
\hline
\end{tabular}

$n=$ number of strains tested. 
Table 4 - Chi-square test for trend applied to E. coli resistance rates (\%) to various antimicrobials in 4 surveyed years (2000 to 2003).

\begin{tabular}{lrrrrcc}
\hline & $\mathbf{2 0 0 0}$ & $\mathbf{2 0 0 1}$ & $\mathbf{2 0 0 2}$ & $\mathbf{2 0 0 3}$ & $\chi^{\mathbf{2}}$ for Trend & p Value \\
\hline Ampicillin & 44.3 & 41.0 & 42.8 & 45.0 & 0.243 & 0.6 \\
Ciprofloxacin & 9.3 & 10.8 & 13.5 & 14.0 & 77.366 & $<0.0001$ \\
Nitrofurantoin & 3.1 & 2.4 & 3.5 & 2.7 & 0.550 & 0.5 \\
Norfloxacin & 9.4 & 10.9 & 13.7 & 14.0 & 76.736 & $<0.0001$ \\
Tetracycline & 35.0 & 29.9 & 29.6 & 28.9 & 98.600 & $<0.0001$ \\
Trimeth-sulfa & 33.5 & 33.0 & 34.3 & 33.6 & 0.384 & 0.5 \\
Nalidixic acid & 13.1 & 14.3 & 17.2 & 17.4 & 51.520 & $<0.0001$ \\
\hline
\end{tabular}

In the present study, among the 22,679 E. coli tested, the resistance rate to trimethoprim-sulfamethoxazole was $33.7 \%$. Additionally, $11.9 \%$ of the E. coli were resistant to ciprofloxacin, $12.0 \%$ to norfloxacin and $15.5 \%$ to nalidixic acid. Amongst the gram-positive isolates, E. faecalis presented considerable resistance to ciprofloxacin $(16.1 \%)$. Although with a different methodology, the present study demonstrated similar E. coli resistance rates when compared to the Hummers-Pradier clinical study (15); nevertheless E. faecalis showed a marked difference in the resistance rate to ciprofloxacin (82.8\%) if compared to the same study (15). There were also significant differences in the E. coli resistance rates to trimethoprimsulfamethoxazole in comparison to recent studies from other environments, which reported rates from $15-25 \%$ in a surveillance (6) and a clinical study (16), and from around $2-15 \%$ on isolates with a single crossresistance in another surveillance study (17). This study showed that nitrofurantoin was very active against isolates of E. coli and E. faecalis but not against K. pneumoniae, similar to other studies $(6,15$ $18)$.

Furthermore, the present study detected that resistance rates of $\mathrm{E}$. coli, $\mathrm{P}$. mirabilis and $\mathrm{K}$. pneumoniae to ceftriaxone were $0.3 \%, 0.4 \%$ and $1.7 \%$, respectively. A possible explanation to this fact might be the presence of Extended Spectrum BetaLactamases (ESBL) in these strains (all of them in the age groups over 4 years old - data not shown). This finding suggests the existence of non-commu- nity acquired infections among the population under surveillance (possibly but not exclusively nursing homes and home-care facilities), which may have tests performed in the present centre. It is worth to mention that ceftriaxone is not the best screening drug for ESBLs and this was not the objective of this study. However, this is a relevant finding, since it might indicate that infections with these types of strains are being detected in outpatients in Brazil - although further confirmation and stratification for risk factors are needed.

As seen in Table-3, E. coli resistance rates for ciprofloxacin and norfloxacin were $24.3 \%$ and $24.4 \%$ respectively in the age group of $\geq 60$ years old, while it was below $8 \%$ for both drugs in all other age groups. It was also noticed a variation of E. coli resistance rates to ampicillin and trimethoprimsulfamethoxazole between age groups, with higher rates seen in the age groups below 13 years old. As for tetracycline, E. coli resistance rates ranged from $26.6 \%$ to $37.3 \%$, showing generally more elevated resistance levels among all age groups; although with a particular higher resistance rate seen in the age group $\geq 60$ years old. Finally, nitrofurantoin showed low resistance rates in all age groups, with a higher rate observed for this drug in the group $\geq 60$ years old (5.9\%).

Additionally, the chi-square test for trend shown in Table-4 has demonstrated apparently stable resistance rates to nitrofurantoin, ampicillin, and trimethoprim-sulfamethoxazole $(\mathrm{P}>0.5)$, though ex- 
tremely higher rates to the latter two drugs. On the other hand, increasing resistance rates to ciprofloxacin, norfloxacin, and nalidixic acid $(\mathrm{P}<$ 0.0001 ) have been detected, with approximately $9.0 \%$ resistance to ciprofloxacin and norfloxacin in 2000 and $14.0 \%$ in 2003. Surprisingly, an ordered decline of the resistance rate to tetracycline was observed, with $35.0 \%$ in 2000 and $28.9 \%$ in 2003 ( $\mathrm{P}<0.0001$ ). These data may have many interpretations, among them a possible changing pattern of antimicrobial use in the population within the period surveyed, with increased use of fluoroquinolones and decreased use of other drugs, particularly in the older population. However, if these findings are due to other factors different from the suggested ones, such as clonal dissemination, as described by Manges et al. (19), or methodological limits, remains to be determined by further studies.

A final remark must be made on the use of routine antimicrobial susceptibility testing data based on breakpoint concentrations and the limits imposed by the method. Although this has been used before and is acceptable (15), detailed shifts or subtle differences in susceptibility rates may be lost by its use, particularly for agents where susceptible isolates may cluster near a breakpoint. However, it is the opinion of the authors that this would be more problematic in cross-sectional studies, where trends cannot be analyzed and, thus, the same method cannot be compared throughout a certain period.

\section{CONCLUSIONS}

Elevated resistance rates in E. coli from urinary tract infections in outpatients to ampicillin, trimethoprim-sulfamethoxazole, and tetracycline preclude the use of these drugs for empirical treatment in the environment evaluated for all age groups. Instead, nitrofurantoin should be used as empirical therapy for primary, non-complicated urinary tract infections (except in patients with impaired renal function) in outpatients of the greater São Paulo area, Brazil. Thus, there is sufficient indication showing that the E. coli resistance trend for fluoroquinolones is increasing, and although not clarified by this study, possibly linked to higher use of this drug class either by the individual and/or by the population. Due to this trend, this class should be used with caution, particularly if no microbiological documentation is available. At last, resistance prevalence studies may be a useful tool for guiding antimicrobial therapy and helping curb resistance due to selective pressure in community-acquired infections, especially UTI.

\section{CONFLICT OF INTEREST}

\author{
None declared.
}

\section{REFERENCES}

1. Gupta K, Hooton TM, Stamm WE: Increasing antimicrobial resistance and the management of uncomplicated community-acquired urinary tract infections. Ann Intern Med. 2001; 135: 41-50.

2. Zhanel GG, Karlowsky JA, Harding GK, Carrie A, Mazzulli T, Low DE, et al.: A Canadian national surveillance study of urinary tract isolates from outpatients: comparison of the activities of trimethoprimsulfamethoxazole, ampicillin, mecillinam, nitrofurantoin, and ciprofloxacin. The Canadian Urinary Isolate Study Group. Antimicrob Agents Chemother. 2000; 44: 1089-92.

3. Goldstein FW: Antibiotic susceptibility of bacterial strains isolated from patients with community-acquired urinary tract infections in France. Eur J Clin Microbiol Infect Dis. 2000; 19: 112-7.

4. Bukharie HA, Saeed IM: Antimicrobial Resistance Among Pathogens Causing Acute Uncomplicated UTIs. Infect Med. 2001; 18: 358-62.

5. Gupta K, Scholes D, Stamm WE: Increasing prevalence of antimicrobial resistance among uropathogens causing acute uncomplicated cystitis in women. JAMA. 1999; 281: 736-8.

6. Jones RN, Kugler KC, Pfaller MA, Winokur PL: Characteristics of pathogens causing urinary tract infections in hospitals in North America: results from the SENTRY Antimicrobial Surveillance Program, 1997. Diagn Microbiol Infect Dis. 1999; 35: 55-63.

7. Jones RN: Detection of emerging resistance patterns within longitudinal surveillance systems: data sensitivity and microbial susceptibility. MYSTIC Advisory 
Board. Meropenem Yearly Susceptibility Test Information Collection. J Antimicrob Chemother. 2000; 46 Suppl T2: 1-8.

8. Masterton RG: Surveillance studies: how can they help the management of infection? J Antimicrob Chemother. 2000; 46 Suppl T2:53-8.

9. National Committee for Clinical Laboratory Standards. Methods for dilution antimicrobial susceptibility tests for bacteria that grow aerobically, Wayne, Pa; 2000. NCCLS Document M100-S10.

10. National Committee for Clinical Laboratory Standards. Methods for dilution antimicrobial susceptibility tests for bacteria that grow aerobically, Wayne, Pa; 2001. NCCLS Document M100-S11.

11. National Committee for Clinical Laboratory Standards. Methods for dilution antimicrobial susceptibility tests for bacteria that grow aerobically, Wayne, Pa; 2002. NCCLS Document M100-S12.

12. National Committee for Clinical Laboratory Standards. Methods for dilution antimicrobial susceptibility tests for bacteria that grow aerobically, Wayne, Pa; 2003. NCCLS Document M100-S13.

13. Altman DG: Practical Statistics for Medical Research. London, Chapman \& Hall. 1991, pp. 611.

14. Foxman B, Barlow R, D'Arcy H, Gillespie B, Sobel JD: Urinary tract infection: self-reported incidence and associated costs. Ann Epidemiol. 2000; 10: 50915.

15. Hummers-Pradier E, Koch M, Ohse AM, Heizmann WR, Kochen MM: Antibiotic resistance of urinary pathogens in female general practice patients. Scand J Infect Dis. 2005; 37: 256-61.

16. Alos JI, Serrano MG, Gomez-Garces JL, Perianes J: Antibiotic resistance of Escherichia coli from community-acquired urinary tract infections in relation to demographic and clinical data. Clin Microbiol Infect. 2005; 11: 199-203.

17. Kahlmeter G, Menday P: Cross-resistance and associated resistance in 2478 Escherichia coli isolates from the Pan-European ECO.SENS Project surveying the antimicrobial susceptibility of pathogens from uncomplicated urinary tract infections. J Antimicrob Chemother. 2003; 52: 128-31.

18. Farrell DJ, Morrissey I, De Rubeis D, Robbins M, Felmingham D: A UK multicentre study of the antimicrobial susceptibility of bacterial pathogens causing urinary tract infection. J Infect. 2003; 46: 94-100.

19. Manges AR, Johnson JR, Foxman B, O'Bryan TT, Fullerton KE, Riley LW: Widespread distribution of urinary tract infections caused by a multidrug-resistant Escherichia coli clonal group. N Engl J Med. 2001; 345: 1007-13.

\author{
Correspondence address: \\ Dr. Carlos R.V. Kiffer \\ Instituto Fleury, Microbiologia \\ Avenida General Valdomiro de Lima 508 \\ São Paulo, SP, 04344-903, Brazil \\ Fax: + 5511 5014-7601 \\ E-mail: carlos.kiffer@fleury.com.br
}




\section{EDITORIAL COMMENT}

This is an important paper providing susceptibility data on community acquired urinary tract infections (UTI) from a large population. Such information is necessary for working out recommendations on empirical treatment of UTI. Resistant bacterial strains are frequently brought to urology departments by patients with complicated UTI. Therefore, suscep-

\section{EDITORIAL COMMENT}

Urinary tract infections are among the most common community acquired infections. Whereas in nosocomial urinary tract infections surveillance is frequently performed around the world or even mandatory in some countries, data on outpatients' urinary tract infections are still scarce. This study investigated more than 37,000 urinary isolates of outpatients in the Sao Paulo urban area. Antibiotics such as trimethoprim/ sulfamethoxazole exhibited tibility data from primary health care is also important information for urologists.

From a European perspective, it is interesting to note that frequently used drugs like mecillinam and fosfomycin trometamol still seem to be unused therapeutic weapons.

Dr. Truls E. Bjerklund Johansen

Chair, European Society for Infections in Urology Telemark Central Hospital, Urology Section Porsgrunn, Norway E-mail:tebj@sthf.no

high resistant rates, which precludes the use of this substance as primary empiric treatment for outpatient urinary tract infections. Additionally there was a significant increase in fluoroquinolone resistance over the last years to levels where treatment failures might occur in significant cases. Studies like this will help to improve and tailor empiric antibiotic treatment for urinary tract infections in the area of Sao Paulo.
Dr. Florian M. E. Wagenlehner
Department of Urology
Justus-Liebig-University Giessen
Giessen, Germany
E-mail: wagenlehner@aol.com 